\title{
Mastering Model Uncertainty by Transfer from Virtual to Real System
}

\author{
Nicolas Brötz $\left.{ }^{(}\right)$, Manuel Rexer, and Peter F. Pelz \\ Technische Universität Darmstadt, Chair of Fluid Systems, \\ 64287 Darmstadt, Germany \\ \{nicolas.broetz, manuel.rexer, peter.pelz\}@fst.tu-darmstadt.de \\ https://www.fst.tu-darmstadt.de
}

\begin{abstract}
Two chassis components were developed at the Technische Universität Darmstadt that are used to isolate the body and to reduce wheel load fluctuation.

The frequency responses of the components were identified with a stochastic foot point excitation in a hardware-in-the-loop (HiL) simulation environment at the hydropulser. The modelling of the transmission behaviour influence of the testing machine on the frequency response was approximately represented with a time delay of $10 \mathrm{~ms}$ in the frequency range up to $25 \mathrm{~Hz}$. This is considered by a Padé approximation. It can be seen that the dynamics of the testing machine have an influence on the wheel load fluctuation and the body acceleration, especially in the natural frequency of the unsprung mass. Therefor, the HiL stability is analysed by mapping the poles of the system in the complex plane, influenced by the time delay and virtual damping.

This paper presents the transfer from virtual to real quarter car to quantify the model uncertainty of the component, since the time delay impact does not occur in the real quarter car test rig. The base point excitation directly is provided by the testing machine and not like in the case of the HiL test rig, the compression of the spring damper calculated in the real-time simulation.
\end{abstract}

Keywords: Test rig $\cdot$ Stability $\cdot$ Model uncertainty $\cdot$ Time delay • Active Air Spring · Fluid Dynamic Vibration Absorber

\section{Introduction}

Developing new products or technologies is always at a high level of risk. To minimise the latter, it is essential evaluating the function and quality, cf. Pelz et al. [1], of the innovation as early as possible in the development process and to examine the interaction with the overall system. Early evaluation also corresponds to agile product development. One method to implement agile product development in the design process is HiL [2]. In HiL experiments, the newly developed component is integrated into a virtual system, thus enabling an accelerated or shortened development time.

(C) The Author(s) 2021

P. F. Pelz and P. Groche (Eds.): ICUME 2021, LNME, pp. 35-44, 2021.

https://doi.org/10.1007/978-3-030-77256-7_4 
HiL was first used for aerospace applications [3]. Since 1980 design engineering makes use of HiL for the development of vehicle components [4]. We use HiL to validate the two chassis components developed at the chair of fluid systems at the Technische Universität Darmstadt, (i) the Active Air Spring (AAS) [1] and (ii) the Fluid Dynamic Vibration Absorber (FDVA) [1]. The tests presented in this paper are performed with the FDVA. It intends to reduce wheel load fluctuation by transmitting the vibration energy of the wheel to the structural extension. The hydraulically translated oil mass, pumped by a piston from one chamber to another via ducts, represents the inductance. The inductance is connected to a compliance, thus a coil spring [5]. Figure 1 shows the FDVA on the right hand side. We will not focus on the component but on the two test rigs used to validate it in a dynamic system.

HiL is not without uncertainty, especially uncertainty due to boundary conditions and time delay appear. In this paper, we highlight the time delay. Batterbee et al. came to the conclusion that HiL test rig dynamics is to degrade performance results of a damper at higher frequencies [6]. Research is done for variability in time delays by Guillo-Sensano et al. [7]. Also, the interface location for HiL with time delay is an object of research by Terkovics et al. [8]. All the research is done because the time delay leads to an instability of the system. Therefore, the need of time delay reduction or compensation is high. Osaki et al. use a simple compensation strategy by adding a virtual damping to the system [9].

In this paper we have a look at the model uncertainty of the FDVA in a dynamic quarter car system. We look at the impact of our HiL stability with time delay of $10 \mathrm{~ms}$ [10]. To analyze the stability we evaluate the poles of the system with varying virtual damping and time delay. Knowing the effects of time delay, we discuss the problem of time delay compensation and describe the possibility of quantifying the FDVA's model uncertainty by finally introducing a quarter car test rig that does not have the time delay.

\section{Hardware-in-the-Loop Test Rig with Time Delay}

A common method to validate a hardware component in a complex system is to use HiL. The benefit for that is a reduced effort in manufacturing the system the component is used in. We build such a test rig, see Fig. 1, to validate the simulation models of suspension components and the components themselves.

The HiL test rig consists of connected real-time simulation and hardware [10]. In the real-time simulation a quarter car is simulated, that is reduced to a wheel mass $m_{\mathrm{w}}$, a body mass $m_{\mathrm{b}}$ and a linear tire model with stiffness $k_{\mathrm{t}}$ and damping $b_{\mathrm{t}}$. The quarter car model is excited via the input variable, the road excitation $z_{\mathrm{r}}$, mapping the drive over a federal highway at $100 \mathrm{~km} / \mathrm{h}$. The outputs of the quarter car model are the body and wheel displacement $z_{\mathrm{b}}, z_{\mathrm{w}}$. Both values are subtracted and fed into the controller of the uni axial test rig. This moves the hydraulic cylinder to stimulate the FDVA in a deflection controlled manner. The signal transmission is impacted with a time delay that we discuss in the 
following. At the top of the FDVA we measure the force $F$ and feed it back to the quarter car model. Besides this force $F$ a virtual damper with the damping constant $\tilde{b}_{\mathrm{b}}$ acts between the wheel and body mass. Table 1 shows the parameters of the quarter car.

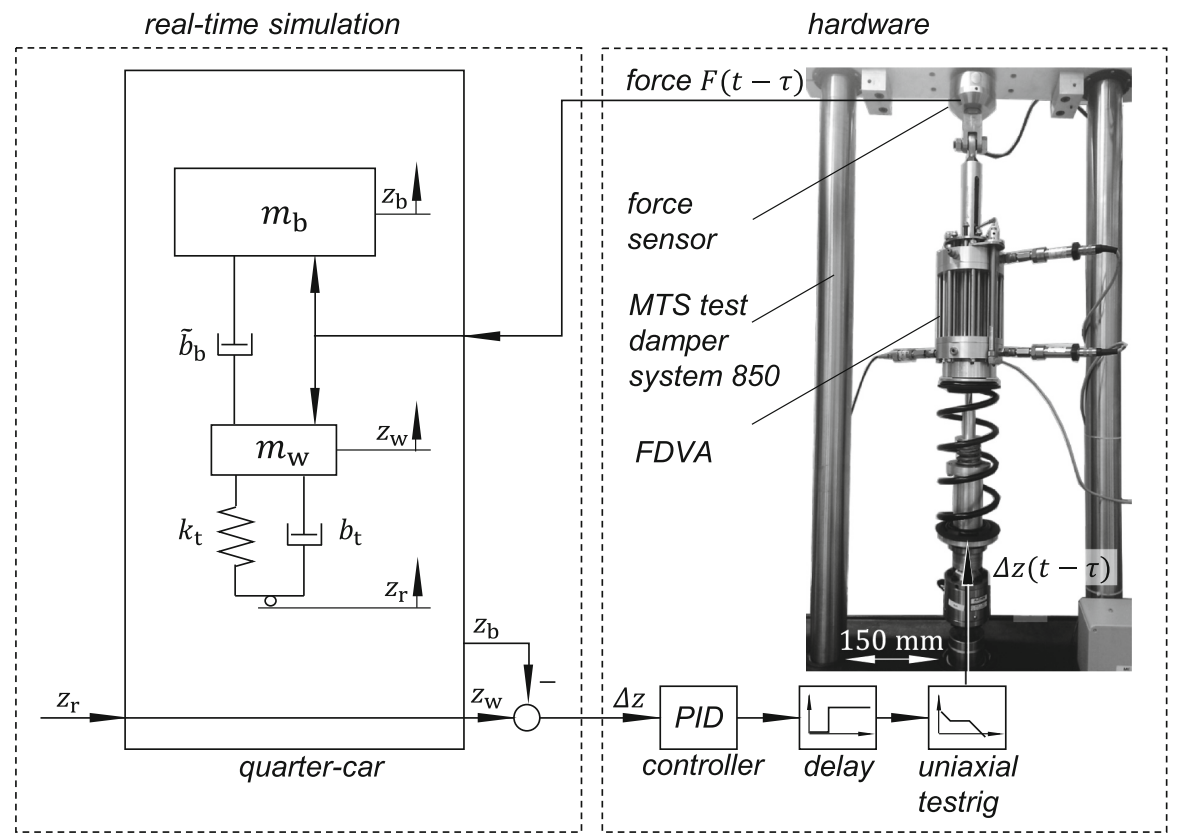

Fig. 1. Hardware-in-the-Loop test rig with quarter car simulation and MTS test damper system with integrated Fluid Dynamic Vibration Absorber

The validated model of the FDVA $[5,11]$ is now tested in a dynamic system environment. The measurement results are evaluated by the amplifications of the frequency response, which is common for suspension components. The virtual damping $\tilde{b}_{\mathrm{b}}$ was implemented to test the AAS that needed an additional damper. The FDVA should be tested without any additional virtual damping $\tilde{b}_{\mathrm{b}}$ because the FDVA on its own can reduce the amplification of the wheel movement in the wheel eigenfrequency [5]. But reducing the virtual body damping from $1140 \mathrm{Ns} / \mathrm{m}$ to $700 \mathrm{Ns} / \mathrm{m}$ gets the system unstable. Having a look at the frequency response in Fig. 2, the measurement and simulation of the FDVA in the HiL test rig differ. You can find the amplification between wheel load and road excitation on the left and on the right you see the amplification between body acceleration and road excitation. The amplifications of wheel eigenfrequency at $13 \mathrm{~Hz}$ differ by more than $400 \%$. Thus, we have to eliminate the source leading to this model uncertainty at the wheel eigenfrequency. Therefore we have a look at the stability of the system. 
Table 1. Quarter car parameters

\begin{tabular}{l|l|l}
\hline Parameter & Variable & Value \\
\hline Body mass & $m_{\mathrm{b}}$ & $290 \mathrm{~kg}$ \\
\hline Wheel mass & $m_{\mathrm{w}}$ & $40 \mathrm{~kg}$ \\
\hline Body stiffness & $k_{\mathrm{b}}$ & $31500 \mathrm{~N} / \mathrm{m}$ \\
\hline Body damping & $b_{\mathrm{b}}$ & $1140 \mathrm{Ns} / \mathrm{m}$ \\
\hline Tyre stiffness & $k_{\mathrm{t}}$ & $200000 \mathrm{~N} / \mathrm{m}$ \\
\hline Tyre damping & $b_{\mathrm{t}}$ & $100 \mathrm{Ns} / \mathrm{m}$ \\
\hline Time delay & $\tau$ & $10 \mathrm{~ms}$ \\
\hline
\end{tabular}
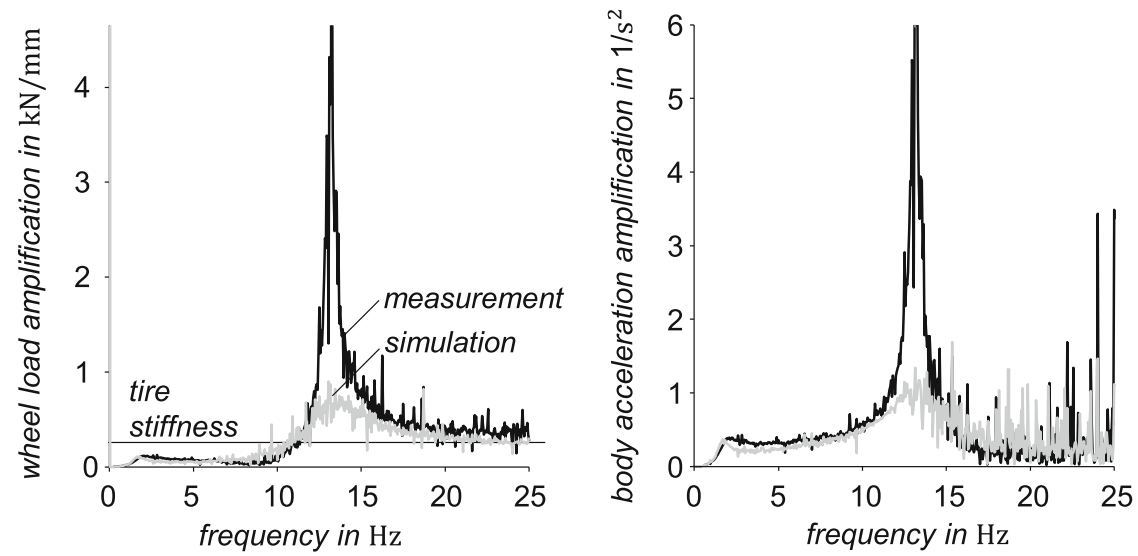

Fig. 2. HiL quarter car results with FDVA driving over a federal highway at $100 \mathrm{~km} / \mathrm{h}$. The FDVA with two opened ducts has an eigenfrequency at $8 \mathrm{~Hz}$

\subsection{HiL Stability}

The HiL test rig controller is a black box. The PID parameters can be changed, but there is no possibility to detect the different time delays inside of it or even reduce them. Based on this we have to accept that there is an overall time delay $\tau=10 \mathrm{~ms}$. To analyse the impact of the time delay $\tau$ and the virtual damping $\tilde{b}_{\mathrm{b}}$ we investigate the equations of motion for the quarter car model

$$
\begin{aligned}
& m_{\mathrm{b}} \ddot{z}_{\mathrm{b}}(t)=F(t-\tau)+\tilde{b}_{\mathrm{b}}\left[\dot{z}_{\mathrm{w}}(t)-\dot{z}_{\mathrm{b}}(t)\right] \\
& m_{\mathrm{w}} \ddot{z}_{\mathrm{w}}(t)= \\
& \quad k_{\mathrm{t}}\left[z_{\mathrm{r}}(t)-z_{\mathrm{w}}(t)\right]+b_{\mathrm{t}}\left[\dot{z}_{\mathrm{r}}(t)-\dot{z}_{\mathrm{w}}(t)\right] \\
& \quad-F(t-\tau)-\tilde{b}_{\mathrm{b}}\left[\dot{z}_{\mathrm{w}}(t)-\dot{z}_{\mathrm{b}}(t)\right] \\
& F(t-\tau)= \\
& k_{\mathrm{h}}\left[z_{\mathrm{w}}(t-\tau)-z_{\mathrm{b}}(t-\tau)\right]+b_{\mathrm{b}}\left[\dot{z}_{\mathrm{w}}(t-\tau)-\dot{z}_{\mathrm{b}}(t-\tau)\right] .
\end{aligned}
$$

We transform Eqs. (1) and (2) in first order differential equations and use a linearized force, see Eq. (3), to evaluate general impacts. In MATLAB we analyse the poles of the resulting state space model, see Fig. 3. The first and second pole 
of the HiL system are shown. By increasing the virtual damping constant $\tilde{b}_{\mathrm{b}}$ the poles move left and the system gets more stable, but we want to reduce the virtual damping to investigate the real damping. By adding more time delay all poles move to the right and the real term of one pole gets positive and gets the system unstable.

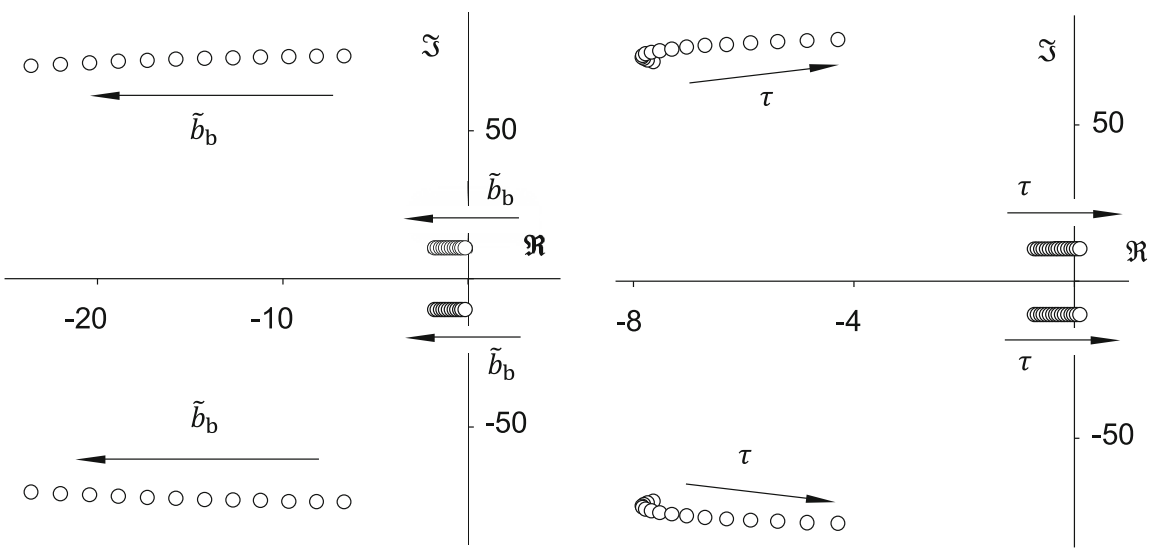

Fig. 3. HiL system poles depending on the simulated damping constant (left) and on the time delay (right)

Thus, the HiL system gets unstable at a time delay $\tau=10 \mathrm{~ms}$. The HiL system instability is shown in Fig. 4. The Bode and Nyquist plot show that next to the wheel eigenfrequency at $13 \mathrm{~Hz}$ where the phase reaches $-180 \mathrm{deg}$, the magnitude is above $0 \mathrm{~dB}$. For better visualisation the Nyquist Plot shows the Nyquist locus circles clockwise around the point $[-1,0]$. Therefor we have a negative damping that leads to the amplification at the wheel eigenfrequency shown in Fig. 2.
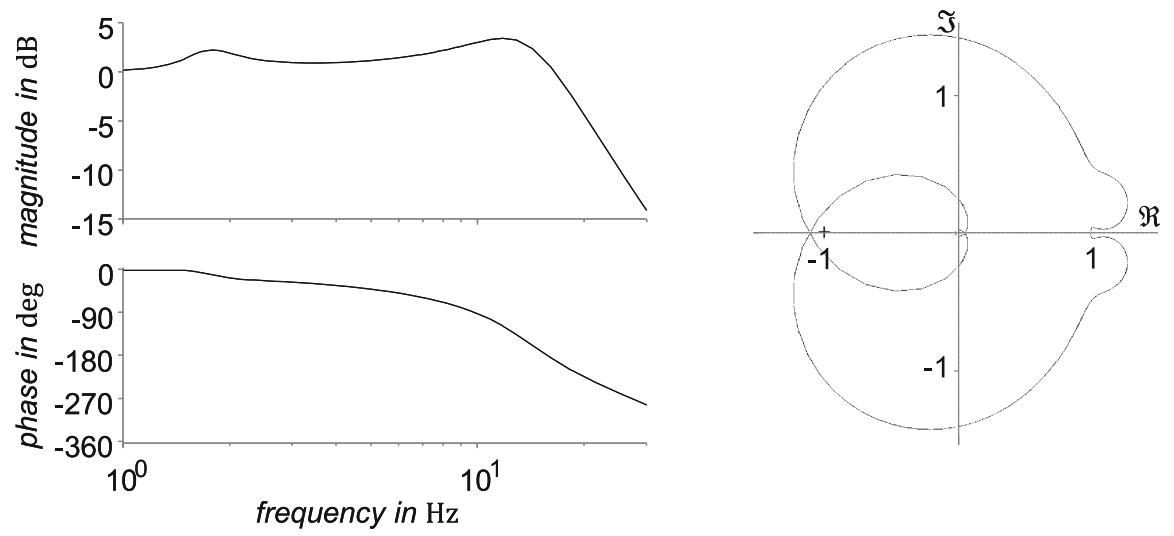

Fig. 4. Bode diagram (left) and Nyquist plot (right) for the unstable HiL quarter car with input $z_{\mathrm{r}}$ and output $z_{\mathrm{w}}$ at a time delay $\tau=10 \mathrm{~ms}$ 


\subsection{Time Delay Compensation Problems for Model Validation}

Time delay must be compensated to stabilize a HiL system. If we are testing a damper, there should be no virtual damping investigating real damping, but in our case, the system gets unstable. The simple compensation strategy by adding virtual damping is possible to stabilise the system, but this is no way to get lose of the time delay. For non-linear systems like the FDVA a state controller with a state monitoring is necessary to compensate the time delay. The state controller itself needs a simulation model of the nonlinear component and thus implies the model uncertainty we want to investigate. Therefore, the only way to quantify model uncertainty with measurement data is a system with no time delay. For this purpose, we have set up a hardware quarter car test rig, witch will be explained in the following section.

\section{Quarter Car Test Rig}

The developed quarter car test rig consists of a servo-hydraulic foot point excitation and a load frame on which the masses of the test rig can be guided by means of height-adjustable transverse control arms. This setup makes it possible to integrate a variety of axle kinematics into the test rig. The servo-hydraulic foot point excitation system from Form+Test includes a power supply, a valve block and a cylinder that can be moved with displacement or force control. The power unit has an output of $22 \mathrm{~kW}$ and provides a pressure of $280 \mathrm{bar}$. The installed cylinder has a maximum force of $25 \mathrm{kN}$ at a maximum velocity of $0.7 \mathrm{~m} / \mathrm{s}$. The cylinder stroke is limited to $250 \mathrm{~mm}$, which can be measured via the integrated stroke sensor, cf. Table 2. Furthermore, a force sensor is mounted on the piston rod to determine the wheel load. All measured variables of the system are transmitted to the measurement data recording. An interface of the controller is available for specifying the cylinder path.

Figure 5 shows the test rig with integrated FDVA. The kinematics used is the Modular Active Spring Damper System (MAFDS) developed as a demonstrator in the Collaborative Research Centre 805. Pelz et al. give a detailed description and its possibilities [1]. The MAFDS consists of a bar structure and three joint modules that absorb all lateral forces of the suspension system. Two coil springs connected in parallel are used as a wheel with the stiffness $k_{\mathrm{t}}$ specified in Table 1 . The masses of the system can be flexibly adjusted. For this purpose, steel plates with $10 \mathrm{~kg}$ each are mounted on a support frame, so that the required body mass is achieved. Steel weights of different masses are also installed for the wheel. Therefore, the system can be easily tuned. The centres of gravity of each degree of freedom are located centrally above the cylinder.

Table 2 shows the sensors available for measuring the state quantities. The wheel force as well as all accelerations and displacements of the excitation and the two masses are captured. The velocity is determined by a combination of the derivative of the displacement and integration of the acceleration. Laser distance sensors with digital interfaces are used for precise measurement and lownoise signal transmission. The acceleration sensors are three similar piezoelectric 
sensors. The data acquisition is carried out by means of a MicroLabBox from dSpace, where it is also possible to specify any excitation $z_{\mathrm{r}}$ of the system. Since this is a real-time simulation environment, complex state estimators such as Kalman filters or controllers for active and semi-active systems can be realised.

Table 2. Sensors of the quarter car test rig

\begin{tabular}{l|l|l|l|l}
\hline Sensor & Label & Variable & Range & Linearity \\
\hline Body acceleration & IMI Sensors 626B02 & $\ddot{z}_{\mathrm{b}}$ & $\pm 98 \mathrm{~m} / \mathrm{s}^{2}$ & $0.98 \mathrm{~m} / \mathrm{s}^{2}$ \\
\hline Wheel acceleration & IMI Sensors 626B02 & $\ddot{z}_{\mathrm{w}}$ & $\pm 98 \mathrm{~m} / \mathrm{s}^{2}$ & $0.98 \mathrm{~m} / \mathrm{s}^{2}$ \\
\hline Road acceleration & IMI Sensors 626B02 & $\ddot{z}_{\mathrm{r}}$ & $\pm 98 \mathrm{~m} / \mathrm{s}^{2}$ & $0.98 \mathrm{~m} / \mathrm{s}^{2}$ \\
\hline Wheel force & GBR Serie-dr & $F_{\mathrm{w}}$ & $\pm 10 \mathrm{kN}$ & $10 \mathrm{~N}$ \\
\hline Road displacement & MTS RH & $z_{\mathrm{r}}$ & $0 \ldots 275 \mathrm{~mm}$ & $0.04 \mathrm{~mm}$ \\
\hline Tire deflection & RIFTEK RF605-65/250 & $\Delta z_{\mathrm{w}}$ & $65 \ldots 315 \mathrm{~mm}$ & $0.25 \mathrm{~mm}$ \\
\hline Suspension deflection & RIFTEK RF605-65/250 & $\Delta z_{\mathrm{b}}$ & $5 \ldots 315 \mathrm{~mm}$ & $0.25 \mathrm{~mm}$ \\
\hline
\end{tabular}

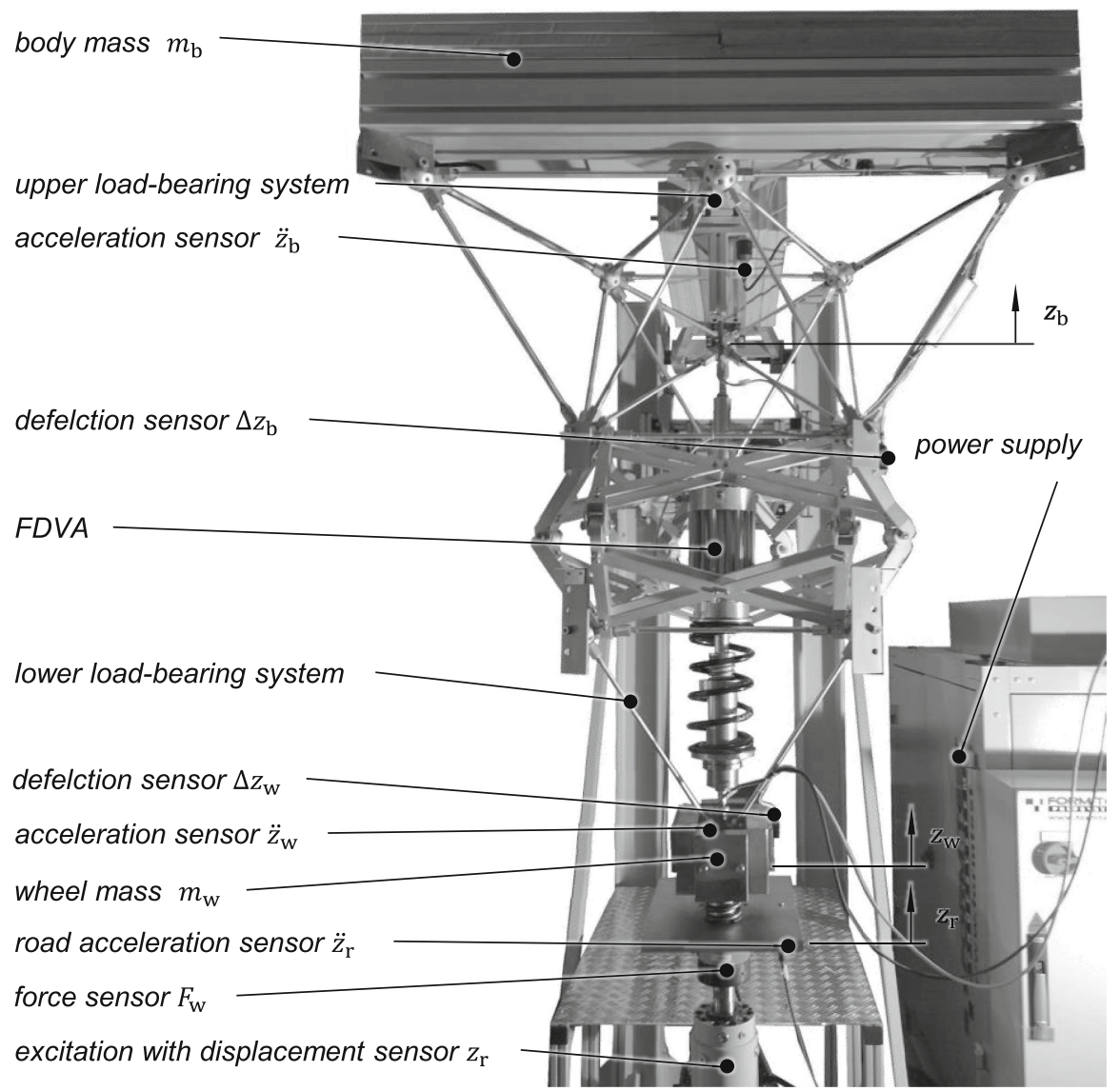

Fig. 5. Quarter car test rig with servo hydraulic foot point excitation and installed sensors 


\subsection{FDVA Results in the Quarter Car Test Rig}

When we now use this quarter car test rig to validate the FDVA model in the dynamic quarter car we can perform tests without an additional body damper. The actual qualitative comparison between measurement and simulation for the quarter car test rig is possible, see Fig. 6 . There is a good agreement between the two lines in the body acceleration amplification. The wheel load amplification shows a difference at higher frequencies, resulting from the mass of the connection of the force sensor and the wheel spring. This mass has an inertia that leads to data uncertainty. With the use of the tire deflection sensor and measurement of the tire spring stiffness the wheel load $F_{\mathrm{w}, \mathrm{c}}=\Delta z_{\mathrm{w}} k_{\mathrm{t}}$ can be calculated. The wheel load amplification of this soft sensor calculated wheel load shows a good agreement to the simulation, see Fig. 6 . The model uncertainty is reduced to a minimum, because there is no simulated model for the hardware quarter car. Thus the basis to validate our FDVA model in a dynamic system is given with this test rig.
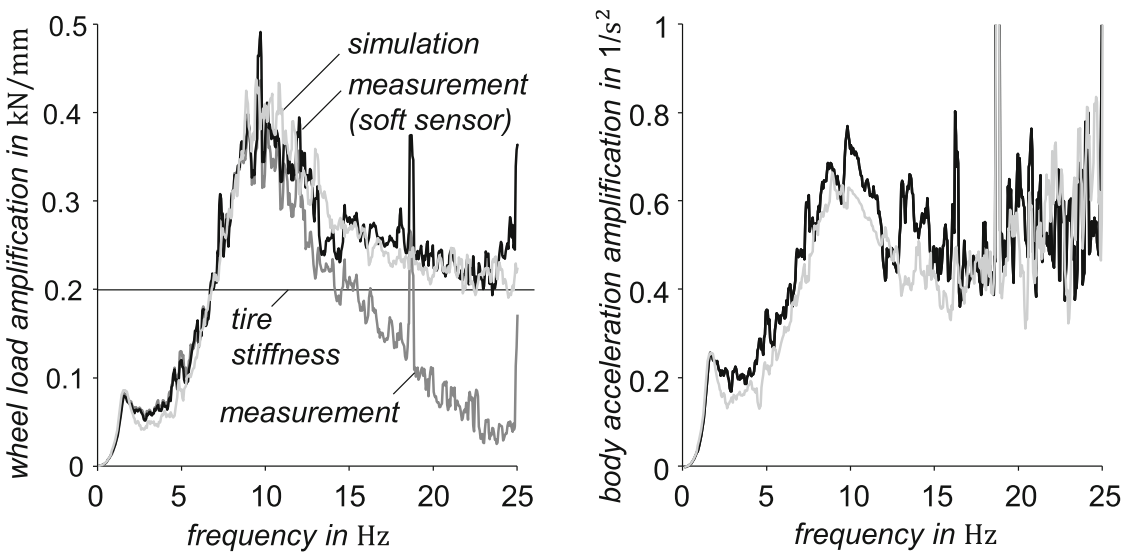

Fig. 6. FDVA results in the quarter car test rig driving over a federal highway at $100 \mathrm{~km} / \mathrm{h}$. The FDVA with two opened ducts has an eigenfrequency at $8 \mathrm{~Hz}$

\section{Conclusion}

We studied the effect of the time delay on the stability of a HiL test rig for the validation of suspension component models. The simulation and measurement of the HiL test rig differ. Model uncertainty in the HiL test rig appears in form of the time delay. Therefore we analysed the poles of the state space model of a quarter car with a linear force feedback. With this understanding of the time delay impact we conclude that especially for non linear components like the Fluid Dynamic Vibration Absorber, there is only the possibility to build a real quarter car test rig to quantify model uncertainty. The quarter car test 
rig in form of hardware is described in detail. We are able to quantify a good agreement between simulation and measurement with this test rig. A detailed quantification of the FDVA model uncertainty by using the real quarter car test rig is part of further research.

Acknowledgement. Funded by the Deutsche Forschungsgemeinschaft (DFG, German Research Foundation) - Project number 57157498 - SFB805.

\section{References}

1. Pelz, P.F., Groche, P., Pfetsch, M.E., Schaeffner, M.: Mastering Uncertainty in Mechanical Engineering. Springer Nature (2021)

2. Hedrich, P., Brötz, N., Pelz, P.F.: Resilient product development - a new approach for controlling uncertainty. Appl. Mech. Mater. 885 (2018)

3. Isermann, R., Jochen, S., Stefan, S.: Hardware-in-the-loop simulation for the design and testing of engine-control systems. Control Eng. Pract. 7(5), 643-653 (1999)

4. Schuette, H., Waeltermann, P.: Hardware-in-the-loop testing of vehicle dynamics controllers-a technical survey. SAE Trans. 593-609 (2005)

5. Brötz, N., Hedrich, P., Pelz, P.F.: Integrated Fluid Dynamic Vibration Absorber for Mobile Applications. Universitätsbibliothek der RWTH Aachen (2018)

6. Batterbee, D.C., Sims, N.D.: Hardware-in-the-loop simulation of magnetorheological dampers for vehicle suspension systems. Proc. Inst. Mech. Eng. Part I: J. Syst. Control Eng. 221(2), 265-278 (2007)

7. Guillo-Sansano, E., Syed, M., Roscoe, A.J., Burt, G., Coffele, F.: Characterization of time delay in power hardware in the loop setups. IEEE Trans. Ind. Electron. 13, $4237(2020)$

8. Terkovics, N., Neild, S.A., Lowenberg, M., Szalai, R., Krauskopf, B.: Substructurability: the effect of interface location on a real-time dynamic substructuring test. Proc. R. Soc. A: Math. Phys. Eng. Sci. 472, 2192 (2016)

9. Osaki, K., Atsushi, K., Masaru, U.: Delay time compensation for a hybrid simulator. Adv. Robot. 24(8-9), 1081-1098 (2010)

10. Lenz, E., Hedrich, P., Pelz, P.F.: Aktive Luftfederung - Modellierung, Regelung und Hardware-in-the-Loop-Experimente. Forschung im Ingenieurwesen 82(3), 171-185 (2018)

11. Brötz, N., Pelz, P.F.: Bayesian Uncertainty Quantification in the Development of a New Vibration Absorber Technology. Model Validation and Uncertainty Quantification, vol. 3, pp. 19-26. Springer, Cham (2020) 
Open Access This chapter is licensed under the terms of the Creative Commons Attribution 4.0 International License (http://creativecommons.org/licenses/by/4.0/), which permits use, sharing, adaptation, distribution and reproduction in any medium or format, as long as you give appropriate credit to the original author(s) and the source, provide a link to the Creative Commons license and indicate if changes were made.

The images or other third party material in this chapter are included in the chapter's Creative Commons license, unless indicated otherwise in a credit line to the material. If material is not included in the chapter's Creative Commons license and your intended use is not permitted by statutory regulation or exceeds the permitted use, you will need to obtain permission directly from the copyright holder. 